\title{
THE BOVINE VIRAL DIARRHOEA VIRUS: A MODEL FOR THE STUDY OF ANTIVIRAL MOLECULES INTERFERING WITH N-GLYCOSYLATION AND FOLDING OF ENVELOPPE GLYCOPROTEIN
}

\author{
D. Durantel ${ }^{1}$, N. Branza-Nichita ${ }^{2}$, S. Durantel ${ }^{1}$, R. A. Dweek ${ }^{3}$, \\ and N. Zitzmann ${ }^{3}$ \\ ${ }^{1}$ Laboratoire des virus hépatiques et pathologies associées \\ INSERM U271, Lyon, France \\ ${ }^{2}$ Institute of Biochemistry \\ Sector 6, Bucharest, Romania \\ ${ }^{3}$ Glycobiology Institute \\ Department of Biochemistry \\ University of Oxford, Oxford, U.K.
}

The current treatment of chronic hepatitis $\mathrm{C}$ combines interferon alpha and ribavirin and is effective in only half of the patients treated. Considerable efforts are being made to develop novel anti-HVC molecules with a better efficacy particularly for refractory patients. Molecules targeting specifically viral activities are the most studied. However, an antiviral strategy based uniquely on the utilisation of this type of molecules is expected to encounter problems caused by the emergence of viral escape mutants, as already widely described for HIV and HBV. Alternative approaches and molecules are needed to complement antiviral strategies based on inhibitors of viral enzyme. Ideally, new molecules should target steps of the viral cycle that are potentially less likely to give rise to resistance. The assembly and morphogenesis of $\mathrm{HCV}$ belong to these yet untargeted steps of the life cycle.

As no cellular system able to support the morphogenesis and secretion of HCV particles is yet available, the bovine viral diarrhoea virus (BVDV), which is phyllogenetically close and shares biological features with $\mathrm{HCV}$, has been used as a surrogate model for the study of antiviral molecules interfering with the $\mathrm{N}$-glycosylation and folding of viral envelope glycoprotein.

We have demonstrated that some analogues of glucose (deoxynojirimycin), also 
generically called iminosugars, are good inhibitors of morphogenesis and prevent viral re-entry by reducing the infectivity of released virions. The mechanism of action has been studied at molecular level for these iminosugars presenting antiviral activity against BVDV. Two different mechanism of action have been defined to explain the whole antiviral effect. DNJ derivatives inhibit host ER a-glucosidases, thus preventing the trimming of 2 glucoses from triglycosylated $\mathrm{N}$-glycans and the subsequent interaction with lectin chaperone. This inhibition results in the misfolding of viral glycoprotein and the subsequent defect in assembly, budding and viral secretion. Moreover, DNJ derivatives induce a diminution of viral infectivity and therefore prevent re-infection of cells by neo-formed particles. This is likely due to the incorporation of non functional envelope glycoprotein complexes.

In conclusion, the BVDV has proven very useful to evaluate the antiviral activity of molecules that inhibit morphogenesis and/or viral entry. The BVDV will remain an interesting model for $\mathrm{HCV}$ while waiting for the development of a cell culture system able to fully propagate the latter. 